\title{
79 Malignant tumours of the middle ear cleft
}

A Adenocarcinoma is the commonest histological type.

B Squamous carcinoma is usually associated with chronic otitis media.

C Intense pain is caused by meningeal involvement.

D Adenocarcinoma is radiosensitive and so surgery is reserved for residual or recurrent disease.

E Radical mastoidectomy is usually performed prior to radiotherapy.

\section{Paragangliomas of the temporal bone}

A Are more aggressive histologically than glomus tumours.

B May present with involvement of the 9th, 10th and 11th cranial nerves.

C Are more common in males.

D With massive intracranial extension are best treated with radiotherapy.

E May occur concurrently with a carotid body tumour.

\section{Osseous disorders of the temporal bone}

A Monostotic fibrous dysplasia is more common than the polyostotic type.

B Osteitis deformans has a rare tendency to sarcomatous change.

C Eosinophilic granuloma produces punched out lesions seen on plain X-rays.

D Osteopetrosis may present as recurring facial palsies. 